\title{
An Exploration of the Range of Noise Intensity That Affects the Membrane Potential of Neurons
}

\author{
Rubin Wang, Guanzheng Wang, and Jinchao Zheng \\ Institute for Cognitive Neurodynamics, School of Science, East China University of Science and Technology, Shanghai 200237, China \\ Correspondence should be addressed to Rubin Wang; rbwang@163.com
}

Received 20 November 2013; Accepted 4 December 2013; Published 12 February 2014

Academic Editor: Jinde Cao

Copyright (C) 2014 Rubin Wang et al. This is an open access article distributed under the Creative Commons Attribution License, which permits unrestricted use, distribution, and reproduction in any medium, provided the original work is properly cited.

Neuronal activity in the human brain occurs in a complex physiologic environment, and noise from all aspects in this physiologic environment affects all aspects of nervous-system function. An essential issue of neural information processing is whether the environmental noise in a neural system can be estimated and quantified in a proper way. In this paper, we calculated the neural energy to estimate the range of critical values of thermal noise intensity that markedly affect the membrane potential and the energy waveform, in order to define such a noisy environment which neuronal activity relies on.

\section{Introduction}

Thermal noise in neural system is critically vital for information processing, because it has a great influence on a variety of aspects of the central nervous system [1-6]. How to evaluate the intensity of such noise has long been a focus for scientists attempting to understand network behaviors and perception [7-11]. So far, estimations of thermal noise intensity in neurons or neural networks in published papers with regard to neural models are generally subjective, lacking a definite basis that is applicable to the range of thermal noise intensity in real systems. Sometimes, authors define thermal noise themselves in order to obtain an ideal result; however, scientific definition of thermal noise and its intensity range is extremely difficult [12-18]. From the viewpoint of quantification, there have been a lot of studies on information coding and the dynamic behaviors of networks in a noisy environment, but these studies have not attempted to answer one of the most basic questions-what principles should be used to choose the thermal noise values in models? Theoretically speaking, there will be deviations between the calculated results and experimental data, and a further discussion on the effectiveness of calculated results is also needed. Taking into account all the considerations above and based on the literature [19-21], in this paper, a range of values of the different membrane potential under the thermal noise condition studied by using the energy method and their corresponding energy waveforms.

We wanted to estimate the range of critical values of noise intensity that is capable of markedly changing the energy waveform. The principal idea of our research was inspired by the fact that since it is impossible to measure the thermal noise intensity at a neuronal level which is great enough to affect neuronal activity in an experiment, and hence, according to the rule of the only corresponding relationship between the membrane potential and its energy function, the range of thermal noise intensity obtained by our neuronal energy function can be estimated to be what it should be in a real neural system. Any kind of membrane potential can be obtained by adjusting the noise intensity, but there is no intrinsic relationship between such a membrane potential and the real neuronal energy. Therefore, we studied the membrane potential starting from the point of view of neuronal energy and observing the type of order of magnitude in noise intensity that can greatly affect the energy function of the membrane potential. As a result, we obtained a range of thermal noise intensity that neurons might have in an actual thermal noisy environment.

It follows that a further discussion of thermal noise intensity range is possible for levels of networks. This part of the research is not only significant for the application of thermal noise intensity when modeling a neuron, but is also 
able to provide an adequate scientific basis for estimating the range of thermal noise intensity in networks and helping to establish neural network models.

Finally, it should be emphasized that we did not consider signal to noise ratio (SNR) that is beyond the scope of this paper.

\section{Methods}

Compared with the traditional simple single neuronal model, a voltage source, a current source, and an inductor are innovatively proposed in the biophysical model presented in this paper, which is shown in Figure 1. The difference in concentration between inner and external ions of neurons creates the electric voltage source that drives the ions. The current source is created by, on one hand, the chemical gradient of ions and, on the other hand, the stimuli from neighboring neurons. Moreover, in the ion channels, the flow of charged particles, such as sodium ions, potassium ions, and calcium ions, forms a loop current which is equivalent to an inductor $L_{m}$, producing self-induction. $C_{m}$ is used to denote the membrane capacitance. $I_{m}$ denotes the total electrical current formed by the inputs of all the neighboring neurons to the $M$ th neuron. $U$ denotes the voltage source. $r_{m}$ and $r_{0 m}$ denote the resistance of the current source $I_{m}$ and voltage source $U$, respectively, showing the wastage produced by the actual sources. Due to the actions of the electric voltage source and the electric current source at different points in the neuron, the membrane resistance is divided into three parts $r_{1 m}, r_{2 m}$, and $r_{3 m}$.

As indicated in Figure 1, the power $P_{m}$ of the $M$ th neuron produced by the voltage source $U_{m}$ and the current source $I_{m}$ is obtained using the formula

$$
P_{m}=U_{m} I_{0 m}+U_{i m} I_{m}
$$

where

$$
\begin{gathered}
U_{m}=r_{0 m} I_{0 m}+r_{1 m} I_{1 m}+L_{m} \dot{I}_{1 m}, \\
I_{0 m}=I_{1 m}-I_{m}+\frac{U_{i m}}{r_{m}}+C_{m} \dot{I}_{1 m}, \\
U_{i m}=C_{m} r_{3 m} \dot{U}_{0 m}+U_{0 m}, \\
L_{m} \dot{I}_{1 m}+r_{1 m} I_{m}=K_{1 m} \dot{U}_{0 m}+K_{2 m} U_{0 m}-r_{2 m} I_{m} .
\end{gathered}
$$

The current source is calculated from the formula

$$
\begin{aligned}
I_{m}= & i_{1 m} \\
& +\sum_{j=1}^{n}\left[i_{0 m}(j-1) \sin \omega_{m}(j-1)\left(t_{j}-t_{j-1}\right)\right] \\
& +i_{0 m}(n) \sin \omega_{m}(n)\left(t-t_{n}\right) \\
& t_{n}<t<t_{n+1}, \quad n=1,2, \ldots, \quad t_{0}=0,
\end{aligned}
$$

where $i_{1 m}$ is the current used to maintain resting potential, $i_{0 m}$ denotes the electric current being produced after the single neuron receives stimuli from all other neighboring neurons

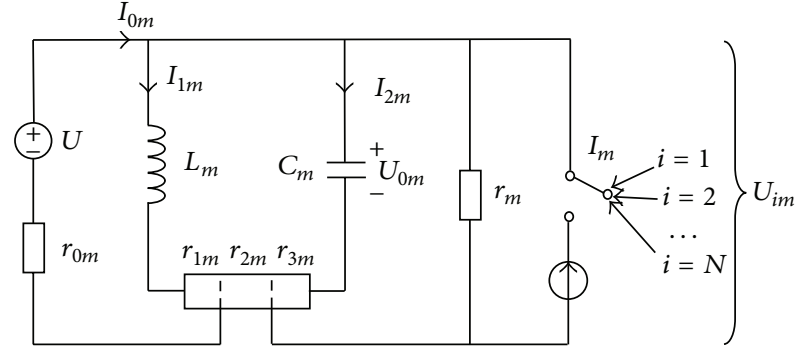

Figure 1: Physical model of the $M$ th neuron with coupling to other neurons.

connected at the subthreshold level, and $\omega_{m}$ denotes the spiking frequency of the action potential, where

$$
K_{1 m}=C_{m}\left(r_{2 m}+r_{3 m}+\frac{r_{2 m} r_{3 m}}{r_{m}}\right), \quad K_{2 m}=1+\frac{r_{2 m}}{r_{m}} .
$$

The solution of (3) is as follows:

$$
\begin{aligned}
I_{1 m}=e^{-a t}\left(K+\frac{1}{L_{m}}(\right. & K_{1 m} U_{0 m} e^{a t}+\left(K_{2 m}-a K_{1 m}\right) \\
& \left.\left.\times \int_{t_{0}}^{t} U_{0 m} e^{a t} d t-r_{2 m} \int_{t_{0}}^{t} I_{m} e^{a t} d t\right)\right),
\end{aligned}
$$

where

$$
a=\frac{r_{1 m}}{L_{m}}, \quad K=I_{1 m}\left(t_{0}\right)-\frac{r_{m}+r_{2 m}}{r_{m} r_{1 m}} U_{0 m}\left(t_{0}\right) .
$$

When (6) satisfies the following conditions:

$$
\begin{gathered}
K_{2 m}-a K_{1 m}=0, \\
L_{m}=\frac{K_{1 m}}{K_{2 m}} r_{1 m}=C_{m} r_{1 m}\left(r_{3 m}+\frac{r_{m} r_{2 m}}{r_{m}+r_{2 m}}\right),
\end{gathered}
$$

we can obtain the following equation:

$$
I_{1 m}=e^{-a t}\left(K+\frac{r_{m} r_{2 m}}{r_{m}+r_{2 m}} U_{0 m} e^{a t}-\frac{r_{2 m}}{L_{m}} \int_{t_{0}}^{t} I_{m} e^{a t} d t\right) .
$$

Inserting (9) and (2) into (1), the power of consumption $P_{m}$ of $m$ th neuron is obtained in the following:

$$
\begin{aligned}
P_{m}= & d_{1 m} \dot{U}_{0 m}^{2}+d_{2 m} \dot{U}_{0 m}+d_{3 m} \dot{U}_{0 m} U_{0 m} \\
& +d_{4 m} U_{0 m}^{2}+d_{5 m} U_{0 m}+d_{6 m}
\end{aligned}
$$

where $d_{1 m}, d_{2 m}, d_{3 m}, d_{4 m}, d_{5 m}$, and $d_{6 m}$ can be obtained from $[20,21]$. 


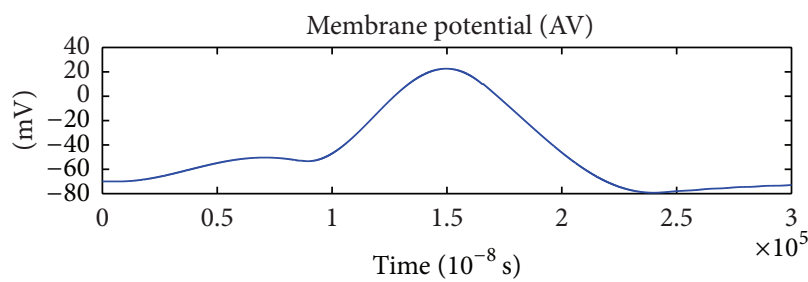

(a)

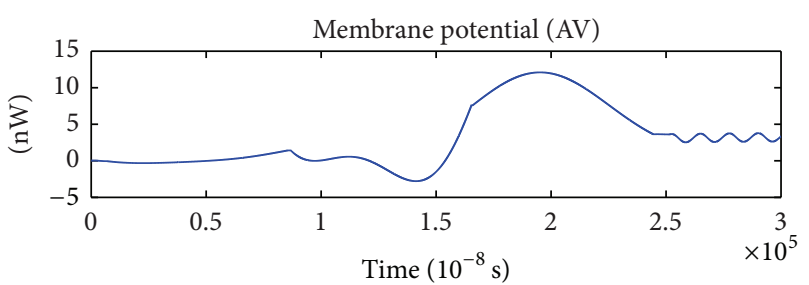

(b)

FIGURE 2: Action potential and its corresponding energy function. $r_{0 m}=0.0001 \Omega, r_{1 m}=0.1 \Omega, r_{2 m}=1000 \Omega, r_{3 m}=0.1 \Omega, r_{m}=1000 \Omega$, $C_{m}=1 \mu \mathrm{F}, L_{m}=50 \mu \mathrm{H}$, and $i_{0 m}=70.7 \mu \mathrm{A}$.

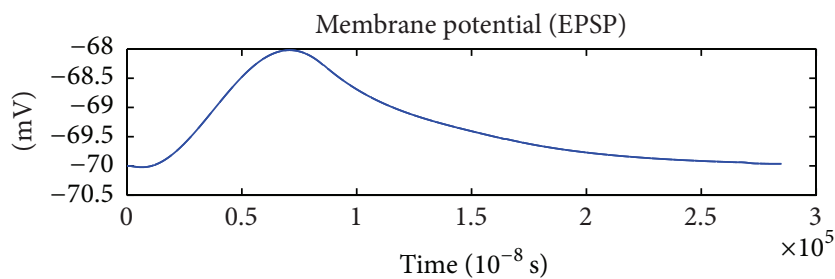

(a)

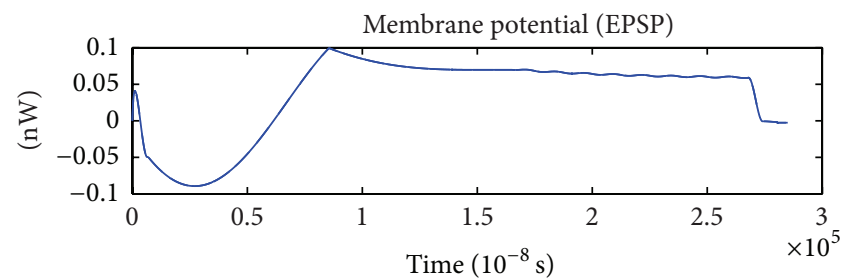

(b)

FIGURE 3: EPSP and its corresponding energy function. $r_{0 m}=0.0001 \Omega, r_{1 m}=0.1 \Omega, r_{2 m}=1000 \Omega, r_{3 m}=0.1 \Omega, r_{m}=1000 \Omega, C_{m}=1 \mu \mathrm{F}$, $L_{m}=50 \mu \mathrm{H}$, and $i_{0 m}=7.155 \mu \mathrm{A}$.

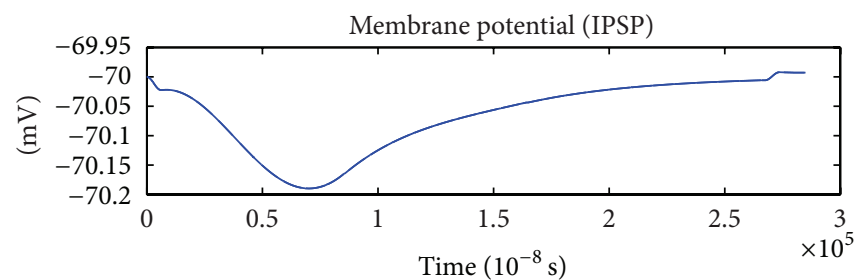

(a)

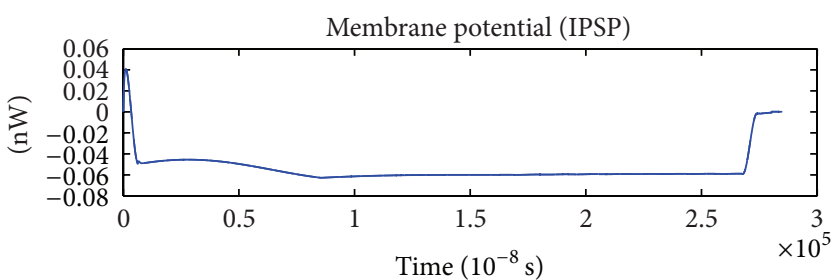

(b)

FIGURE 4: IPSP and its corresponding energy function. $r_{0 m}=0.0001 \Omega, r_{1 m}=0.1 \Omega, r_{2 m}=1000 \Omega, r_{3 m}=0.1 \Omega, r_{m}=1000 \Omega, C_{m}=1 \mu \mathrm{F}$, $L_{m}=50 \mu \mathrm{H}$, and $i_{0 m}=-0.7 \mu \mathrm{A}$.

According to the above equations, we can obtain the solution of the action potential $U_{o m}$ :

$$
\begin{aligned}
U_{0 m}=-\frac{\widehat{g}_{1}}{\lambda_{m}^{2}}- & \frac{\widehat{g}_{2} e^{-a\left(t-t_{n}\right)}}{\lambda_{m}^{2}-a^{2}}-\frac{1}{\lambda_{m}^{2}+\omega_{m}^{2}} \\
\times & \left(\widehat{g}_{3} \sin \left(\omega_{m}(n)\left(t-t_{n}\right)\right)\right. \\
& +\widehat{g}_{4} \cos \left(\omega_{m}(n)\left(t-t_{n}\right)\right) \\
& \times\left(U_{0 m}\left(t_{n}\right)+\frac{\widehat{g}_{1}}{\lambda_{m}^{2}}+\frac{\widehat{g}_{2}}{\lambda_{m}^{2}-a^{2}}\right. \\
& \left.\left.+\frac{\widehat{g}_{4}}{\lambda_{m}^{2}+\omega_{m}^{2}(n)}\right)\right) e^{-\lambda_{m}\left(t-t_{n}\right)} .
\end{aligned}
$$

To clarify our point of view, we present in a straightforward manner the action potential and its neuronal energy function represented by the corresponding power obtained by our proposed method, as shown in Figure 2 .
As indicated in Figure 2, the peak voltage of the action potential is around $25 \mathrm{mV}$ and the peak power of the energy function is around $12 \mathrm{nW}$. The energy waveforms of an excitatory postsynaptic potential (EPSP) and inhibitory postsynaptic potential (IPSP) are shown in Figures 3 and 4, respectively.

In the cerebral cortex, the excitatory neurons comprise $85 \%$ of the neurons, and the remaining neurons are inhibitory [22]. At the subthreshold state, the sum of the power of the EPSP and IPSP of a single neuron is negative ( $\mathrm{PP}=0.0367$ $+(-0.0532)=-0.0165 \mathrm{nW})$. From the overall observation, the total consumed energy is positive $\left(\mathrm{PP}^{\prime}=0.0367 \times 85 \%\right.$ $-0.0532 \times 15 \%=0.0232 \mathrm{nW})$. As seen from the results, the neural system consumes the energy supplied from blood flow, even if the neurons act at a subthreshold state; also as seen from the calculated results, neurons consume more energy at suprathreshold activities than at subthreshold activities. It is reported that a massive number of neurons in subthreshold activities consume only $20 \%$ of the total energy, whereas a small amount of neurons consume more than $80 \%$ due 


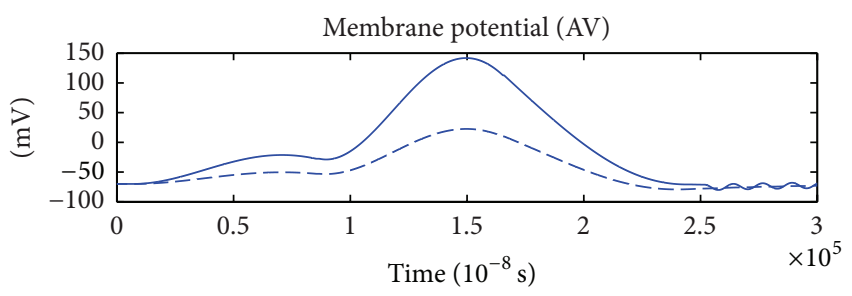

(a)

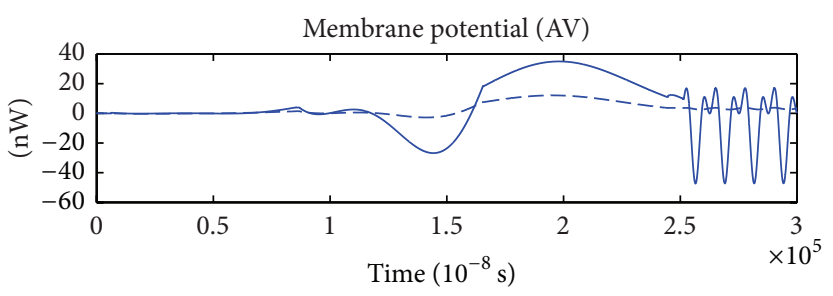

(b)

FIGURE 5: The action potential and its corresponding energy function. $r_{0 m}=0.0001 \Omega, r_{1 m}=0.1 \Omega, r_{2 m}=1000 \Omega, r_{3 m}=0.1 \Omega, r_{m}=1000 \Omega$, $C_{m}=1 \mu \mathrm{F}, L_{m}=50 \mu \mathrm{H}, i_{0 m}=70.7 \mu \mathrm{A}$, and $\mathrm{Q}=1 \times 10^{-4} \mu \mathrm{A}$.

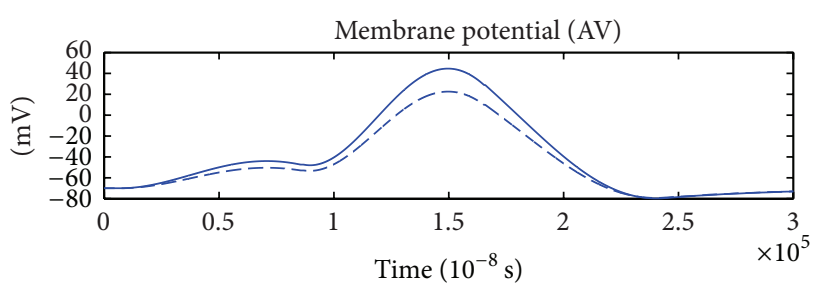

(a)

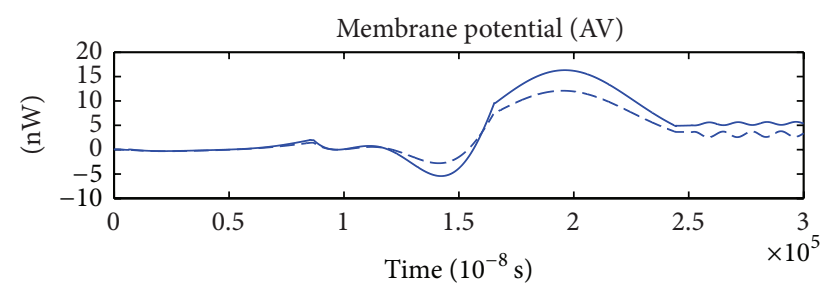

(b)

FIGURE 6: The action potential and its corresponding energy function. $r_{0 m}=0.0001 \Omega, r_{1 m}=0.1 \Omega, r_{2 m}=1000 \Omega, r_{3 m}=0.1 \Omega, r_{m}=1000 \Omega$, $C_{m}=1 \mu \mathrm{F}, L_{m}=50 \mu \mathrm{H}, i_{0 m}=70.7 \mu \mathrm{A}$, and $Q=1 \times 10^{-5} \mu \mathrm{A}$.

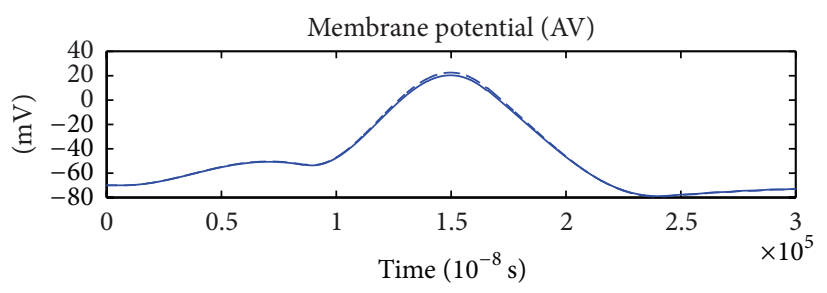

(a)

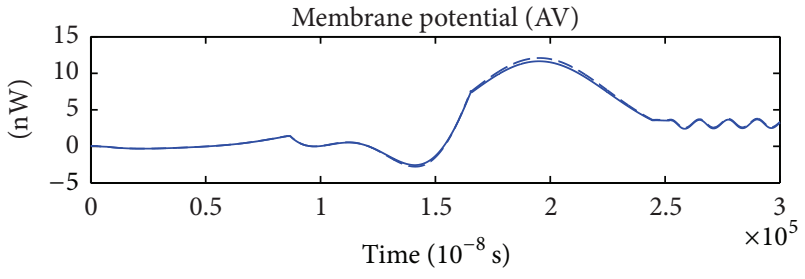

(b)

FIGURE 7: The action potential and its corresponding energy function. $r_{0 m}=0.0001 \Omega, r_{1 m}=0.1 \Omega, r_{2 m}=1000 \Omega, r_{3 m}=0.1 \Omega, r_{m}=1000 \Omega$, $C_{m}=1 \mu \mathrm{F}, L_{m}=50 \mu \mathrm{H}, i_{0 m}=70.7 \mu \mathrm{A}$, and $Q=1 \times 10^{-6} \mu \mathrm{A}$.

to action potential firing [22]. Our results conform to this conclusion.

A single neuron acts under the condition of a neural network. In other words, the interaction among the neurons makes their functional effectiveness emerge. It is in this sense that neuronal activity is performing the process of metabolism in the thermal noisy physiologic environment. To obtain the size of thermal noise intensity of the neuronal activity in the actual environment and to further obtain the noise circumstance under the condition of networks in the brain, we need to first understand the neuronal membrane potential and its corresponding energy function under the condition of no noise interference [19]. Only by obtaining the neuronal energy under an ideal condition can the physiologic influence of thermal noise on the membrane potential and its neuronal energy be better understood. Once that is obtained, it is possible to comprehend the noisy environment in a neural system and evaluate the behaviors of the network in such an environment.

\section{Results}

3.1. The Suprathreshold Membrane Potential and Its Neuronal Energy. Because the signal intensities of AP, EPSP, and IPSP are $7.07 \times 10^{-5} \mu \mathrm{A}, 7.155 \times 10^{-6} \mu \mathrm{A}$, and $7 \times 10^{-7} \mu \mathrm{A}$, so if we superpose thermal noise on the current $i_{0 m}$, when the thermal noise intensity $Q$ is more than $10^{-4} \mu \mathrm{A}$, the influence of thermal noise on membrane potential and its energy function is far greater than that of the current $i_{0 m}$, and when the thermal noise intensity $Q$ is less than $10^{-7} \mu \mathrm{A}$, the influence of thermal noise on membrane potential and its energy function is far weaker than that of $i_{0 m}$. Therefore, the values of thermal noise intensity should be set as $10^{-4} \mu \mathrm{A}, 10^{-5} \mu \mathrm{A}, 10^{-6} \mu \mathrm{A}$, and $10^{-7} \mu \mathrm{A}$, respectively.

After adding thermal noise, the current takes the form

$$
\begin{aligned}
I_{m}= & i_{1 m} \\
& +\sum_{j=1}^{n}\left[\left(i_{0 m}(j-1)+Q\right) \sin \omega_{m}(j-1)\left(t_{j}-t_{j-1}\right)\right] \\
& +\left[i_{0 m}(n)+Q\right] \sin \omega_{m}(n)\left(t-t_{n}\right),
\end{aligned}
$$




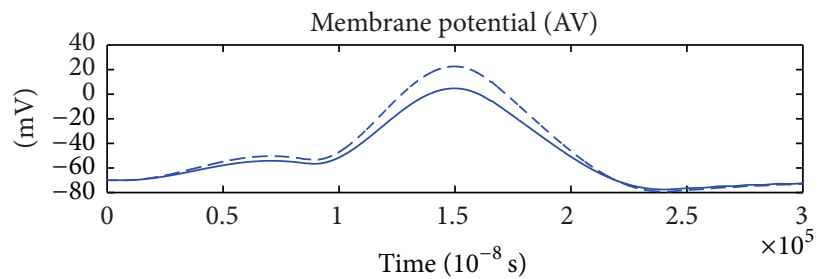

(a)

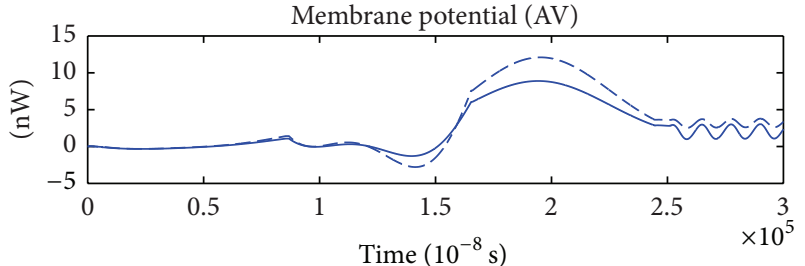

(b)

FIGURE 8: The action potential and its corresponding energy function. $r_{0 m}=0.0001 \Omega, r_{1 m}=0.1 \Omega, r_{2 m}=1000 \Omega, r_{3 m}=0.1 \Omega, r_{m}=1000 \Omega$, $C_{m}=1 \mu \mathrm{F}, L_{m}=50 \mu \mathrm{H}, i_{0 m}=70.7 \mu \mathrm{A}$, and $Q=6 \times 10^{-6} \mu \mathrm{A}$.

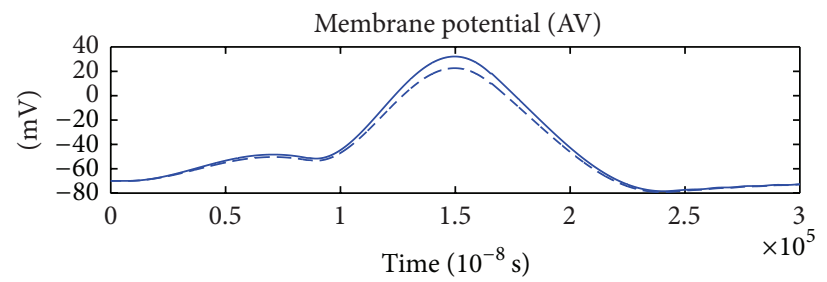

(a)

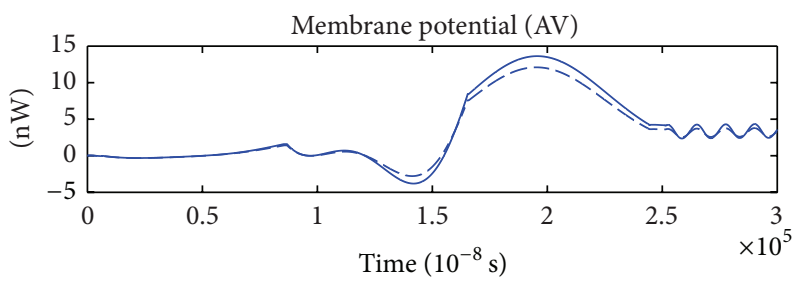

(b)

FIGURE 9: The action potential and its energy function. $r_{0 m}=0.0001 \Omega, r_{1 m}=0.1 \Omega, r_{2 m}=1000 \Omega, r_{3 m}=0.1 \Omega, r_{m}=1000 \Omega, C_{m}=1 \mu \mathrm{F}$, $L_{m}=50 \mu \mathrm{H}, i_{0 m}=70.7 \mu \mathrm{A}$, and $Q=6 \times 10^{-6} \mu \mathrm{A}$.

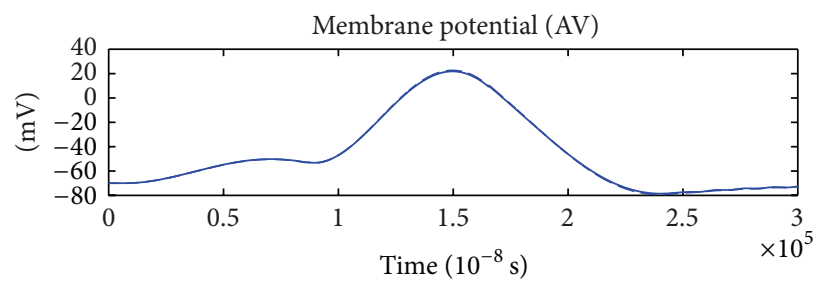

(a)

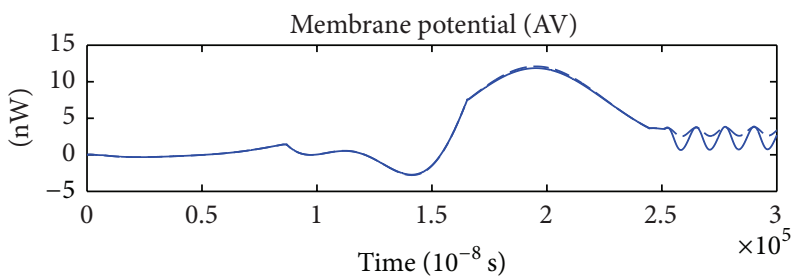

(b)

FIGURE 10: The action potential and its corresponding function. $r_{0 m}=0.0001 \Omega, r_{1 m}=0.1 \Omega, r_{2 m}=1000 \Omega, r_{3 m}=0.1 \Omega, r_{m}=1000 \Omega$, $C_{m}=1 \mu \mathrm{F}, L_{m}=50 \mu \mathrm{H}, i_{0 m}=70.7 \mu \mathrm{A}$, and $Q=6 \times 10^{-6} \mu \mathrm{A}$.

where $Q$ denotes the synaptic noise as Gaussian white noise with the unit $\mu \mathrm{A}$.

The action potential together with its corresponding power plotted by the energy function calculated under the condition of different noise intensity is shown as follows.

In all of Figures 5, 6, 7, 8, 9, and 10, the dotted line represents the case of no noise and the solid line represents the case of noise.

When the noise intensity $Q$ reaches $10^{-4} \mu \mathrm{A}$, the peak of the neuronal membrane potential is around $140 \mathrm{mV}$ and far higher than $25 \mathrm{mV}$ of the situation without noise, while the peak of the energy function is around $40 \mathrm{nW}$ and far higher than the original $12 \mathrm{nW}$. Additionally, on the horizontal axis, between $2.5 \mathrm{~ms}$ and $3 \mathrm{~ms}$, the amplitude of the energy waveform is much larger than the one in the situation without noise. Therefore, it fundamentally changes the action potential and its corresponding energy function under the ideal conditions when $Q$ reaches $10^{-4} \mu \mathrm{A}$.

When the noise intensity $Q$ reaches $10^{-5} \mu \mathrm{A}$, the influence of noise on the action potential deceases, but it is still significant. Here, the membrane potential is around $40 \mathrm{mV}$, whereas the peak of the energy function is around $15 \mathrm{nW}$. As can be seen, such noise intensity still has a great influence on the membrane potential and its corresponding energy waveform.

When the noise intensity $Q$ reaches $10^{-6} \mu \mathrm{A}$, there is hardly any influence on the action potential and its energy function. It is in such a noisy environment that the peaks of the membrane potential and energy waveform are exactly the same with those under the ideal conditions where the peaks of action potential and the energy function are $25 \mathrm{mV}$ and $12 \mathrm{nW}$, respectively. In the presence of noise, the waveforms of the membrane potential and the energy function are nearly the same as without noise.

To summarize, we cannot estimate an accurate value of noise intensity for both the suprathreshold membrane potential and the corresponding neuronal energy. In estimating the range of the critical values of noise intensity, we found that when the noise intensity $Q$ reaches about $6 \times 10^{-6} \mu \mathrm{A}$ (error $\pm 0.5 \times 10^{-6} \mu \mathrm{A}$ ), the noise has great influence on the action potential and its corresponding energy function. 


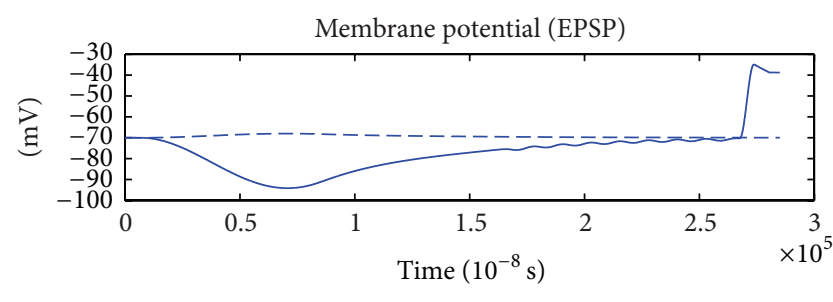

(a)

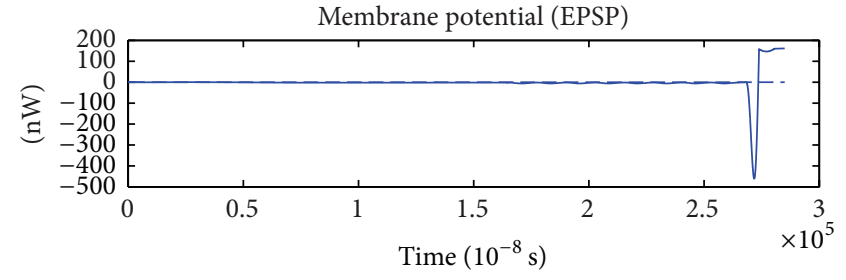

(b)

FIGURE 11: The EPSP and the corresponding energy function. $r_{0 m}=0.0001 \Omega, r_{1 m}=0.1 \Omega, r_{2 m}=1000 \Omega, r_{3 m}=0.1 \Omega, r_{m}=1000 \Omega$, $C_{m}=1 \mu \mathrm{F}, L_{m}=50 \mu \mathrm{H}, i_{0 m}=7.155 \mu \mathrm{A}$, and $Q=1 \times 10^{-4} \mu \mathrm{A}$.

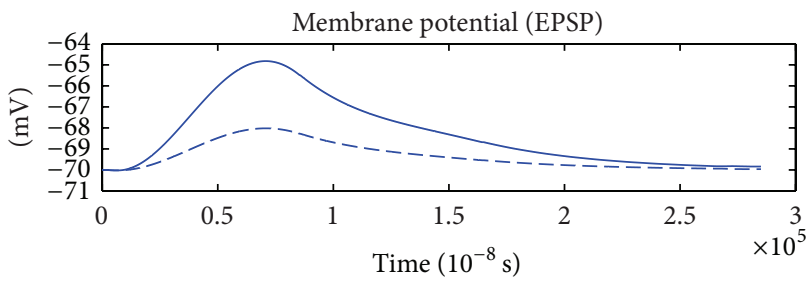

(a)

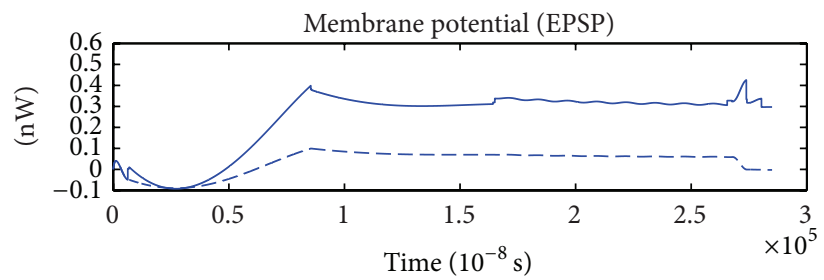

(b)

FIGURE 12: The EPSP and the corresponding energy function. $r_{0 m}=0.0001 \Omega, r_{1 m}=0.1 \Omega, r_{2 m}=1000 \Omega, r_{3 m}=0.1 \Omega, r_{m}=1000 \Omega$, $C_{m}=1 \mu \mathrm{F}, L_{m}=50 \mu \mathrm{H}, i_{0 m}=7.155 \mu \mathrm{A}$, and $Q=1 \times 10^{-5} \mu \mathrm{A}$.

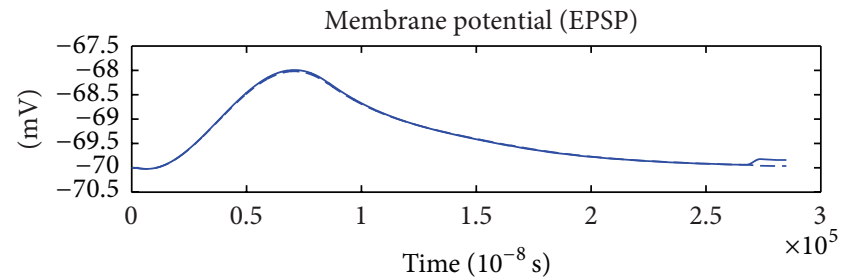

(a)

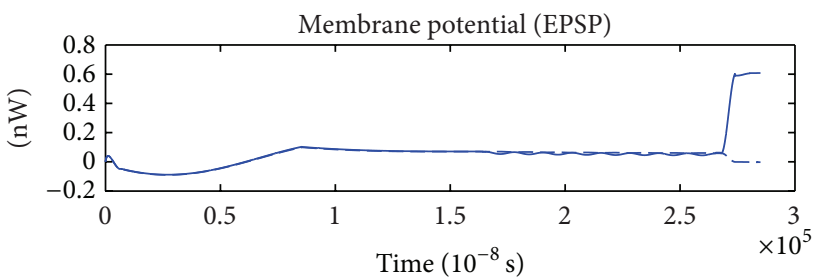

(b)

FIGURE 13: The EPSP and the corresponding energy function. $r_{0 m}=0.0001 \Omega, r_{1 m}=0.1 \Omega, r_{2 m}=1000 \Omega, r_{3 m}=0.1 \Omega, r_{m}=1000 \Omega$, $C_{m}=1 \mu \mathrm{F}, L_{m}=50 \mu \mathrm{H}, i_{0 m}=7.155 \mu \mathrm{A}$, and $Q=1 \times 10^{-6} \mu \mathrm{A}$.

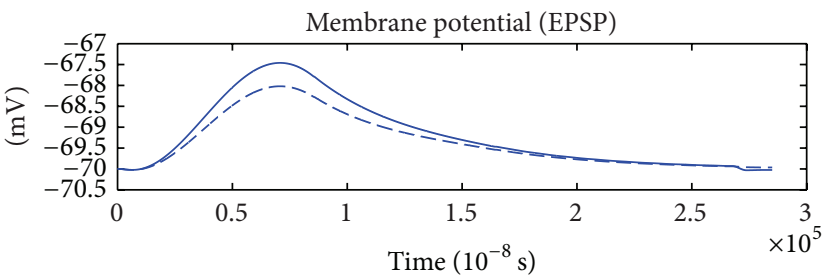

(a)

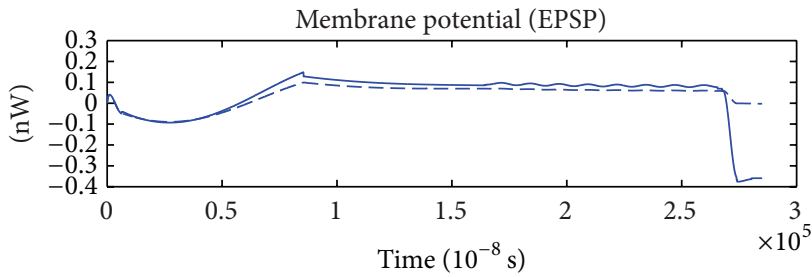

(b)

FIgURE 14: The EPSP and the corresponding energy function. $r_{0 m}=0.0001 \Omega, r_{1 m}=0.1 \Omega, r_{2 m}=1000 \Omega, r_{3 m}=0.1 \Omega, r_{m}=1000 \Omega$, $C_{m}=1 \mu \mathrm{F}, L_{m}=50 \mu \mathrm{H}, i_{0 m}=7.155 \mu \mathrm{A}$, and $\mathrm{Q}=2 \times 10^{-6} \mu \mathrm{A}$.

As shown in Figures 8-10, when the noise intensity $Q$ is $6 \times 10^{-6} \mu \mathrm{A}$, the fluctuation of the membrane potential and the energy function turns out to be very large. The peak of the action potential fluctuates between $10 \mathrm{mV}$ and $40 \mathrm{mV}$, while that of the energy function fluctuates from $10 \mathrm{nW}$ to $15 \mathrm{nW}$. Such noise intensity has great influence on the membrane potential and the energy function, and as a result, the critical value of the noise intensity $Q$ can be assessed at $6 \times 10^{-6} \mu \mathrm{A}$ (error $\pm 0.5 \times 10^{-6} \mu \mathrm{A}$ ).
3.2. Subthreshold Membrane Potential and Its Neuronal Energy. According to the calculated results of Wang et al. [21], we obtained the EPSP and the corresponding energy waveform as well as the IPSP and the corresponding energy waveform by adding noise (Figures 11, 12, 13, 14, 15, 16, 17, 18, $19,20,21,22,23,24$, and 25).

When the level of noise intensity $Q$ reached $1 \times 10^{-4} \mu \mathrm{A}$, it had a great effect on the EPSP and the corresponding energy waveform. Without the effect of noise, we found that the EPSP 


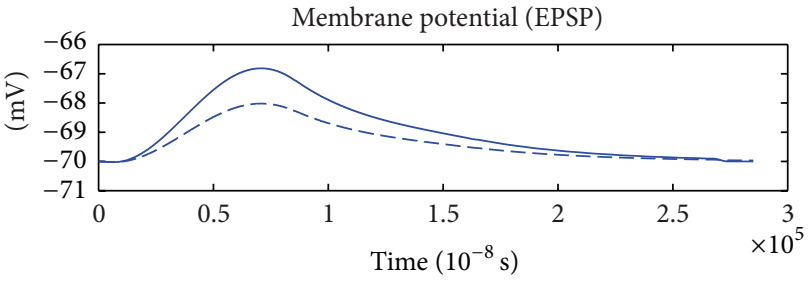

(a)

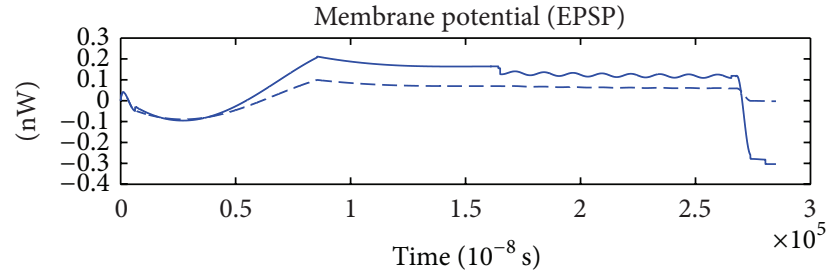

(b)

FIGURE 15: The EPSP and the corresponding energy function. $r_{0 m}=0.0001 \Omega, \quad r_{1 m}=0.1 \Omega, r_{2 m}=1000 \Omega, r_{3 m}=0.1 \Omega, r_{m}=1000 \Omega$, $C_{m}=1 \mu \mathrm{F}, L_{m}=50 \mu \mathrm{H}, i_{0 m}=7.155 \mu \mathrm{A}$, and $\mathrm{Q}=3 \times 10^{-6} \mu \mathrm{A}$.

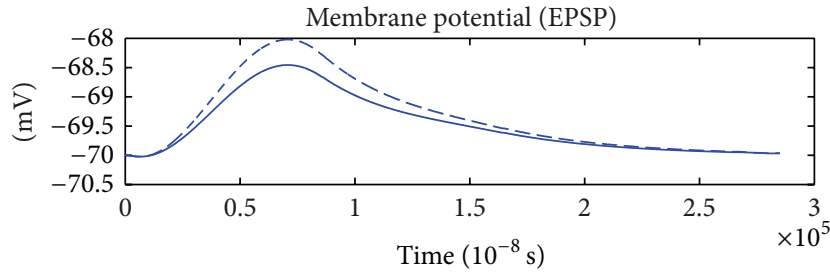

(a)

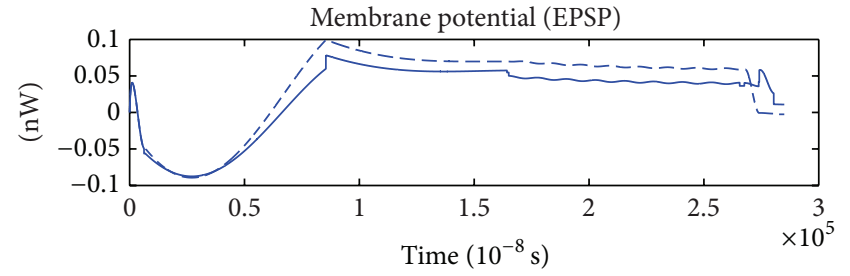

(b)

FIGURE 16: The EPSP and the corresponding energy function. $r_{0 m}=0.0001 \Omega, r_{1 m}=0.1 \Omega, r_{2 m}=1000 \Omega, r_{3 m}=0.1 \Omega, r_{m}=1000 \Omega$, $C_{m}=1 \mu \mathrm{F}, L_{m}=50 \mu \mathrm{H}, i_{0 m}=7.155 \mu \mathrm{A}$, and $\mathrm{Q}=3 \times 10^{-6} \mu \mathrm{A}$.

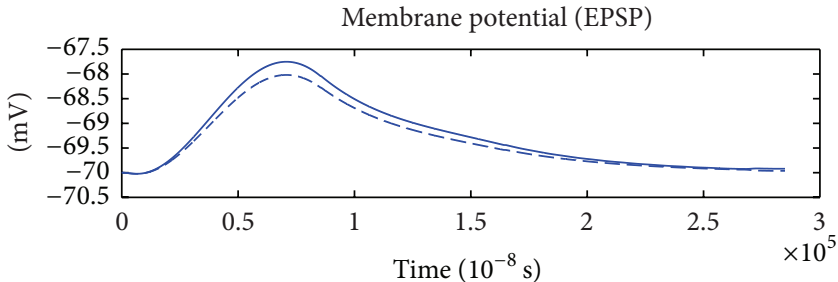

(a)

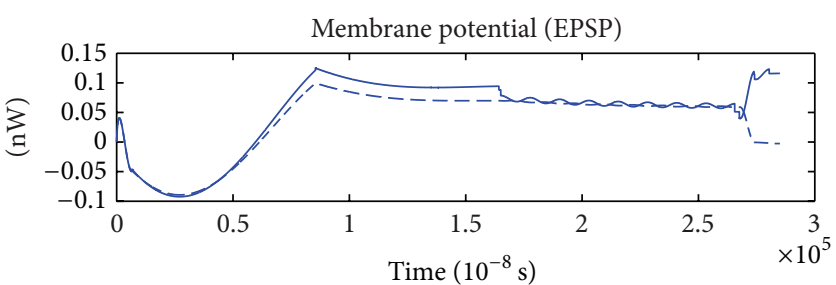

(b)

FIGURE 17: The EPSP and the corresponding energy function. $r_{0 m}=0.0001 \Omega, r_{1 m}=0.1 \Omega, r_{2 m}=1000 \Omega, r_{3 m}=0.1 \Omega, r_{m}=1000 \Omega$, $C_{m}=1 \mu \mathrm{F}, L_{m}=50 \mu \mathrm{H}, i_{0 m}=7.155 \mu \mathrm{A}$, and $Q=3 \times 10^{-6} \mu \mathrm{A}$.

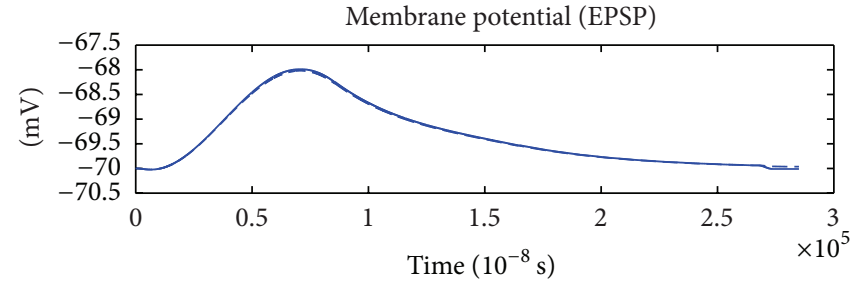

(a)

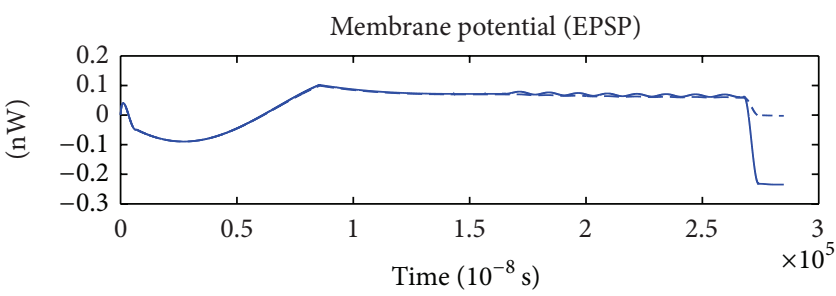

(b)

FIGURE 18: The EPSP and the corresponding energy function. $r_{0 m}=0.0001 \Omega, r_{1 m}=0.1 \Omega, r_{2 m}=1000 \Omega, r_{3 m}=0.1 \Omega, r_{m}=1000 \Omega$, $C_{m}=1 \mu \mathrm{F}, L_{m}=50 \mu \mathrm{H}, i_{0 m}=7.155 \mu \mathrm{A}$, and $\mathrm{Q}=1 \times 10^{-7} \mu \mathrm{A}$.

curve increased first and then decreases with a maximum of $-68 \mathrm{mV}$ and a minimum of $-70 \mathrm{mV}$. The energy function fell first and then rose towards stability, fluctuating in the range of -0.1 to $0.1 \mathrm{nW}$. After adding noise, we found that the EPSP curve fell first and then rose towards a minimum of $-95 \mathrm{mV}$, while the energy function increased first and then decreased towards stability, fluctuating in the range of -10 to $-80 \mathrm{nW}$.
When the level of noise intensity $Q$ reached $1 \times 10^{-5} \mu \mathrm{A}$, it also had a great effect on the EPSP and the corresponding energy waveform. Under an ideal condition without noise, we know that the maximum of the EPSP is about $-68 \mathrm{mV}$ and the peak of the energy function is about $0.1 \mathrm{nW}$. After adding noise, the peak of the EPSP hardly changed, but the oscillatory curve changed sharply in the period from $2.5 \mathrm{~ms}$ to $3 \mathrm{~ms}$. 


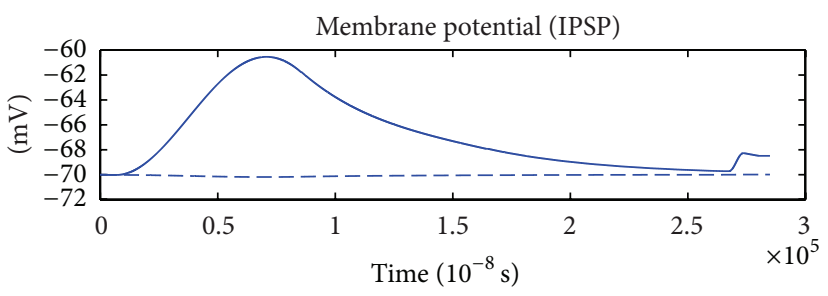

(a)

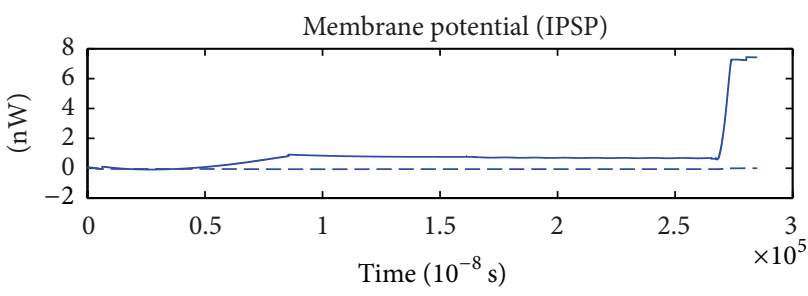

(b)

FIGURE 19: The IPSP and the corresponding energy function. $r_{0 m}=0.0001 \Omega, r_{1 m}=0.1 \Omega, r_{2 m}=1000 \Omega, r_{3 m}=0.1 \Omega, r_{m}=1000 \Omega, C_{m}=1 \mu \mathrm{F}$, $L_{m}=50 \mu \mathrm{H}, i_{0 m}=-0.7 \mu \mathrm{A}$, and $\mathrm{Q}=1 \times 10^{-4} \mu \mathrm{A}$.

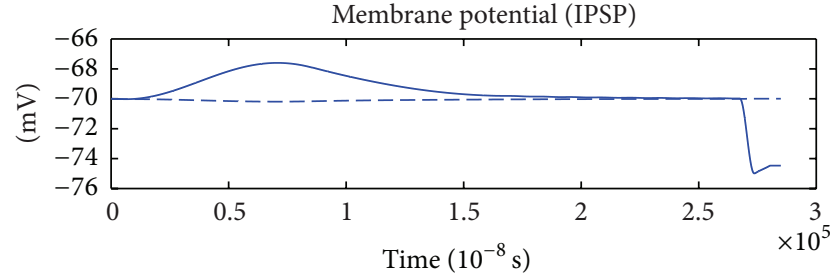

(a)

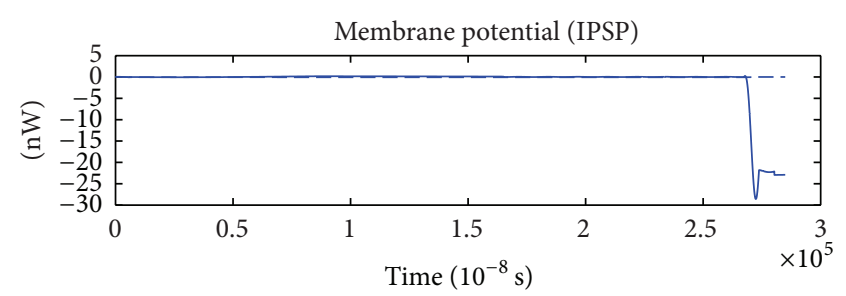

(b)

FIGURE 20: The IPSP and the corresponding energy function. $r_{0 m}=0.0001 \Omega, r_{1 m}=0.1 \Omega, r_{2 m}=1000 \Omega, r_{3 m}=0.1 \Omega, r_{m}=1000 \Omega$, $C_{m}=1 \mu \mathrm{F}, L_{m}=50 \mu \mathrm{H}, i_{0 m}=-0.7 \mu \mathrm{A}$, and $Q=1 \times 10^{-5} \mu \mathrm{A}$.

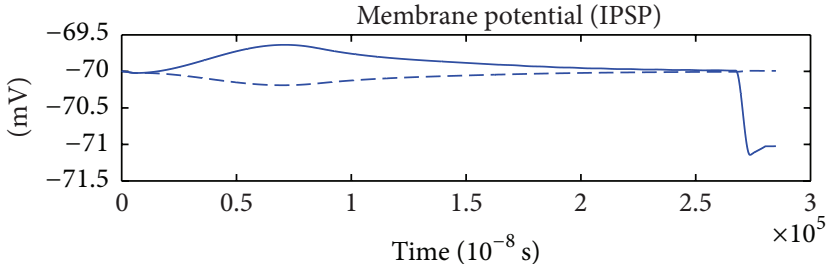

(a)

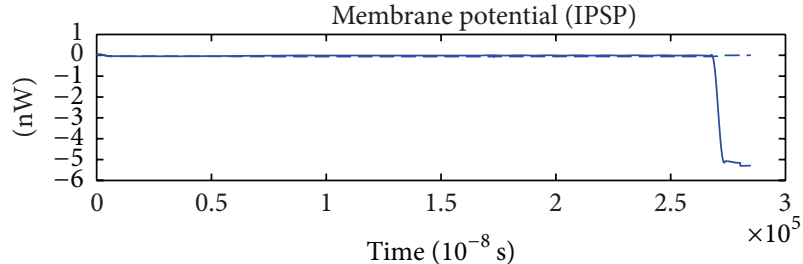

(b)

FIGURE 21: The IPSP and the corresponding energy function. $r_{0 m}=0.0001 \Omega, r_{1 m}=0.1 \Omega, r_{2 m}=1000 \Omega, r_{3 m}=0.1 \Omega, r_{m}=1000 \Omega, C_{m}=1 \mu \mathrm{F}$, $L_{m}=50 \mu \mathrm{H}, i_{0 m}=-0.7 \mu \mathrm{A}$, and $Q=1 \times 10^{-6} \mu \mathrm{A}$.

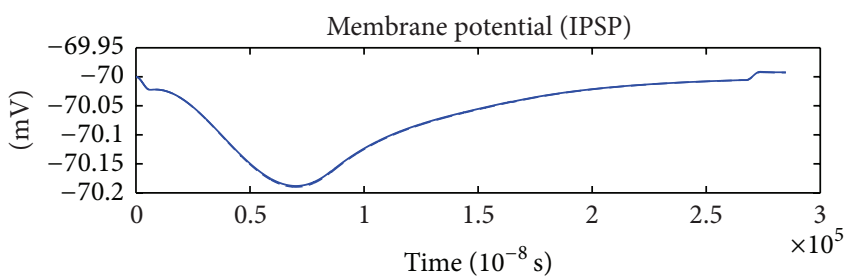

(a)

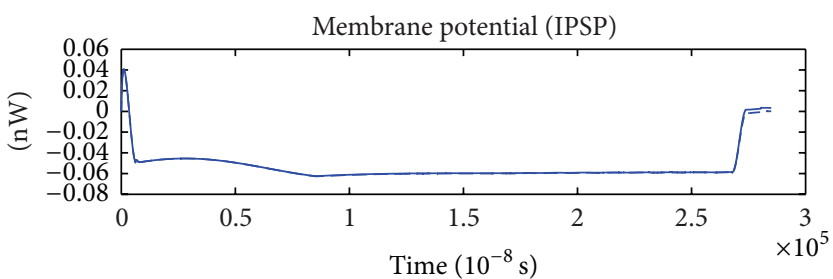

(b)

FIGURE 22: The IPSP and the corresponding energy function. $r_{0 m}=0.0001 \Omega, r_{1 m}=0.1 \Omega, r_{2 m}=1000 \Omega, r_{3 m}=0.1 \Omega, r_{m}=1000 \Omega$, $C_{m}=1 \mu \mathrm{F}, L_{m}=50 \mu \mathrm{H}, i_{0 m}=-0.7 \mu \mathrm{A}$, and $Q=1 \times 10^{-7} \mu \mathrm{A}$.

The oscillatory amplitude of the energy function with noise was more intense than that without noise, varying between $1.5 \mathrm{~ms}$ and $2.5 \mathrm{~ms}$.

When the noise intensity $Q$ reached $1 \times 10^{-6} \mu \mathrm{A}$, it had little effect on the EPSP and the corresponding energy waveform. This figure showing the EPSP and energy function with noise is almost the same with one without noise. We could not estimate the range of the noise intensity, which affects the EPSP's corresponding energy function according to the results shown in Figures 11-13. As a solution, we addressed the problem by adjusting the coefficient of the noise level.

When the level of noise intensity $Q$ reached $2 \times 10^{-6} \mu \mathrm{A}$, it did not have an obvious effect on the EPSP and the corresponding energy waveform. This figure shows the EPSP and energy function with noise is almost the same as one 


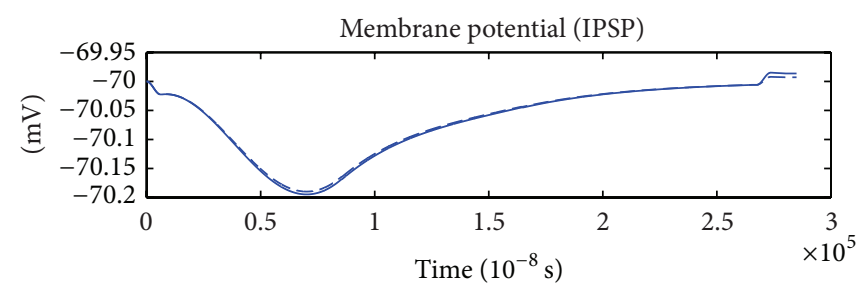

(a)

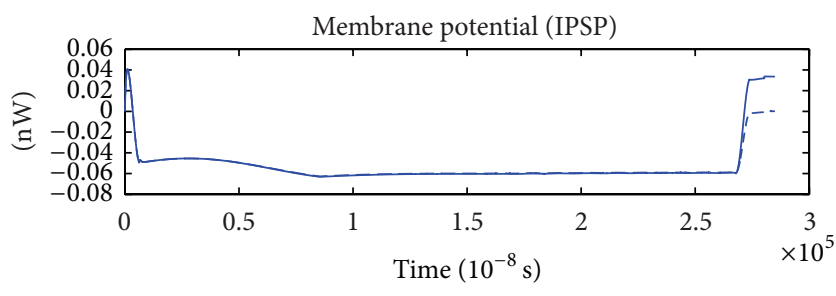

(b)

FIGURE 23: The IPSP and the corresponding energy function. $r_{0 m}=0.0001 \Omega, r_{1 m}=0.1 \Omega, r_{2 m}=1000 \Omega, r_{3 m}=0.1 \Omega, r_{m}=1000 \Omega$, $C_{m}=1 \mu \mathrm{F}, L_{m}=50 \mu \mathrm{H}, i_{0 m}=-0.7 \mu \mathrm{A}$, and $Q=2 \times 10^{-7} \mu \mathrm{A}$.

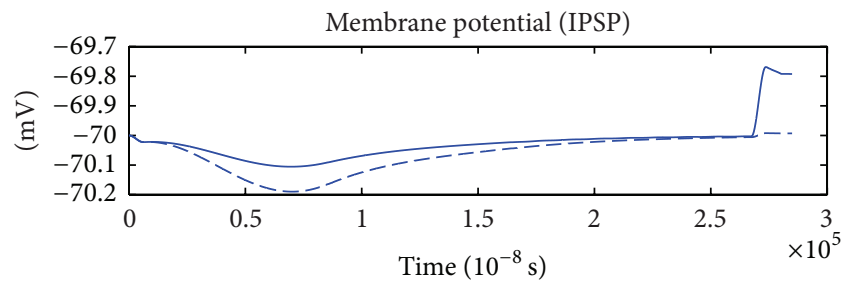

(a)

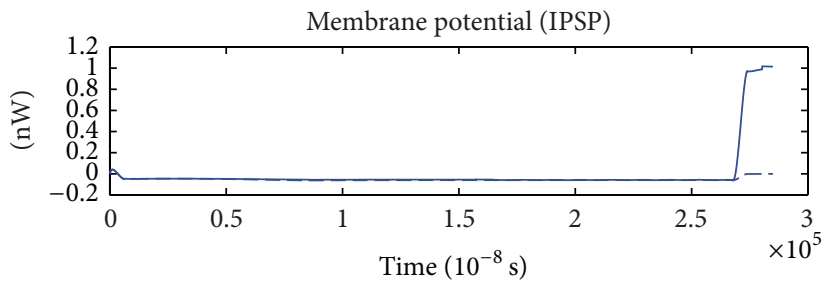

(b)

FIGURE 24: The IPSP and the corresponding energy function. $r_{0 m}=0.0001 \Omega, r_{1 m}=0.1 \Omega, r_{2 m}=1000 \Omega, r_{3 m}=0.1 \Omega, r_{m}=1000 \Omega$, $C_{m}=1 \mu \mathrm{F}, L_{m}=50 \mu \mathrm{H}, i_{0 m}=-0.7 \mu \mathrm{A}$, and $\mathrm{Q}=3 \times 10^{-7} \mu \mathrm{A}$.

without noise. But the energy function with noise is slightly different from that without noise ranging between $1.5 \mathrm{~ms}$ and $2.5 \mathrm{~ms}$.

When the noise intensity $Q$ reached $3 \times 10^{-6} \mu \mathrm{A}$, it had a great effect on the EPSP and the corresponding energy waveform. Taking the noise into account, we found that the EPSP fluctuated in a small interval from $-67 \mathrm{mV}$ to $-69 \mathrm{mV}$, while in the period from $1.5 \mathrm{~ms}$ to $2.5 \mathrm{~ms}$, the energy function with noise had greater fluctuations than that without noise.

When the level of noise $Q$ reached $1 \times 10^{-7} \mu \mathrm{A}$, it had a little effect on the EPSP and the corresponding energy waveform. The figures of the EPSP and energy function with noise are almost the same as those without noise.

In summary, when the noise intensity $Q$ was about $3 \times$ $10^{-6} \mu \mathrm{A}$ (error $\left.\pm 0.5 \times 10^{-6} \mu \mathrm{A}\right)$, the noise begins to have a significant influence on EPSP and its corresponding energy waveform. As a result, the critical value of the noise was assessed at $3 \times 10^{-6} \mu \mathrm{A}\left(\right.$ error $\left.\pm 0.5 \times 10^{-6} \mu \mathrm{A}\right)$.

When the noise intensity $Q$ reached $1 \times 10^{-4} \mu \mathrm{A}$, it had a great effect on the IPSP and the corresponding energy waveform. Without the effect of noise, we found that the IPSP curve fell first and then rose and the energy function curve was almost in the stable state of $-0.04 \mathrm{nW}$. However, after adding noise, we found that the IPSP curve rose first and then fell, while most of the energy function curve was above 0 and there were huge fluctuations of the energy function between $1.5 \mathrm{~ms}$ and $2.5 \mathrm{~ms}$.

When the noise intensity $Q$ reached $1 \times 10^{-5} \mu \mathrm{A}$, it had a critical effect on the IPSP and the corresponding energy waveform. Without the effect of noise, we observed that the IPSP curve fell initially and then rose and the energy function curve was almost in a stable state of $-0.04 \mathrm{nW}$. However, after adding noise, the IPSP curve rose first and then fell and there were huge fluctuations of the energy function between $2.5 \mathrm{~ms}$ and $3 \mathrm{~ms}$.

When the noise intensityQ reached $1 \times 10^{-6} \mu \mathrm{A}$, it had a relatively great effect on the IPSP and the corresponding energy waveform. Without the effect of noise, the IPSP curve fell initially and then rose, while the energy function curve was almost in a stable state of $-0.04 \mathrm{nW}$. After adding noise, the IPSP curve rose first and then fell and there were also huge fluctuations of the energy function from $2.5 \mathrm{~ms}$ to $3 \mathrm{~ms}$.

When the noise intensityQ reached $1 \times 10^{-7} \mu \mathrm{A}$, it had a little effect on the IPSP and the corresponding energy waveform. The figures of the IPSP and energy function with noise are almost the same as those without noise.

We still could not estimate the range of the noise that affects the energy function of the IPSP according to the results shown in Figures 19-22. So as a solution, we addressed it by adjusting the coefficient of the level of noise intensity.

When the noise intensityQ reached $2 \times 10^{-7} \mu \mathrm{A}$, it had a little effect on the IPSP and the corresponding energy waveform. The figures of the IPSP and energy function with noise are almost the same as those without noise.

When the level of noise intensityQ reached $3 \times 10^{-7} \mu \mathrm{A}$, it had a little effect on the IPSP and the corresponding energy waveform. The figures of the IPSP and energy function with noise are almost the same as those without noise.

When the level of noise intensityQ reached about $4 \times$ $10^{-7} \mu \mathrm{A}\left(\right.$ error $\left.\pm 0.5 \times 10^{-7} \mu \mathrm{A}\right)$, the noise began to significantly influence the IPSP and its corresponding energy waveform. Without the effect of noise, the IPSP curve initially fell and then rose; however, with the effect of noise, the IPSP curve did not monotonically decrease in the time interval 


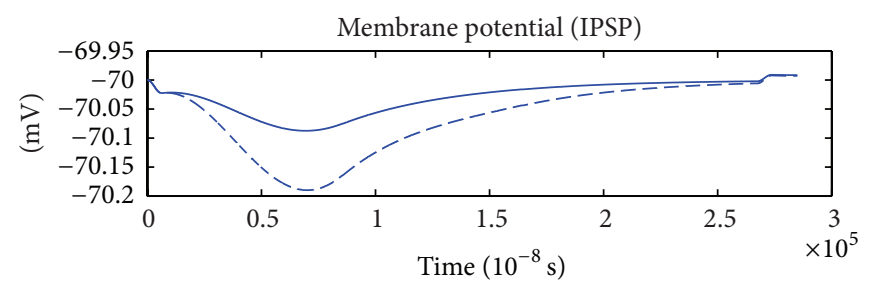

(a)

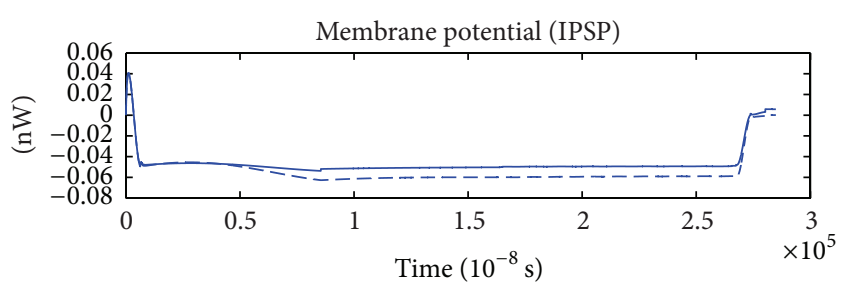

(b)

FIGURE 25: The IPSP and the corresponding energy function. $r_{0 m}=0.0001 \Omega, r_{1 m}=0.1 \Omega, r_{2 m}=1000 \Omega, r_{3 m}=0.1 \Omega, r_{m}=1000 \Omega$, $C_{m}=1 \mu \mathrm{F}, L_{m}=50 \mu \mathrm{H}, i_{0 m}=-0.7 \mu \mathrm{A}$, and $\mathrm{Q}=4 \times 10^{-7} \mu \mathrm{A}$.

from $0 \mathrm{~ms}$ to $1.5 \mathrm{~ms}$, while the energy function began to appear to fluctuate within the interval from $2.5 \mathrm{~ms}$ to $3 \mathrm{~ms}$.

In summary, when the level of noise intensity $Q$ reached about $4 \times 10^{-7} \mu \mathrm{A}$ (error $\pm 0.5 \times 10^{-7} \mu \mathrm{A}$ ), the noise began to have significant influence on the IPSP and its corresponding energy waveform. As a consequence, its critical value was assessed at $4 \times 10^{-7} \mu \mathrm{A}\left(\right.$ error $\left.\pm 0.5 \times 10^{-7} \mu \mathrm{A}\right)$.

\section{Discussion}

In this study, we obtained the action potential, EPSP, IPSP, and their corresponding energy waveforms with thermal noise added to the current. By changing the thermal noise intensity, we found the estimated range of the critical value of thermal noise that can significantly influence the neuronal membrane potential and the corresponding energy waveform. When the thermal noise intensityQ reached about $6 \times$ $10^{-6} \mu \mathrm{A}$ (error $\left.\pm 0.5 \times 10^{-6} \mu \mathrm{A}\right)$, it had a great influence on the action potential and its corresponding energy waveform. When the thermal noise intensity $Q$ reached about $3 \times 10^{-6} \mu \mathrm{A}$ (error $\pm 0.5 \times 10^{-6} \mu \mathrm{A}$ ), it had a great influence on the EPSP and its corresponding energy waveform. When the thermal noise intensityQ reached about $4 \times 10^{-7} \mu \mathrm{A}$ (error $\left.\pm 0.5 \times 10^{-7} \mu \mathrm{A}\right)$, it had a great influence on the IPSP and its corresponding energy waveform. Based on these results, we conclude that noise has less effect on the action potential and its energy waveform than on the EPSP, IPSP, and their energy waveforms, and the thermal noise has less effect on the EPSP and its energy waveform than the IPSP and its waveform. By numerical calculation, we have also obtained the estimated range of the critical value of the noise that has a great effect on the action potential, EPSP, IPSP, and their corresponding energy waveforms. As a result, when we again establish a neural dynamic model in the future, such an estimation of noise intensity will enable us to avoid being subjective in considering the influence of noise on neural firing, so that the calculated results and experimental data will be more consistent. Therefore, the estimation of the thermal noise intensity provides a scientific basis for research on neural information coding in a noisy environment.

The physical model which we used in this paper was proposed by Wang et al. [20, 21] from the viewpoint of energy. Compared with other models, the biggest advantage of this model is that it simplifies the calculation of activity of neuronal firing. Due to the energy coding model's ability to describe mechanisms of brain information processing in biophysical terms, this idea and method can be applied to future research of information encoding in neural ensembles as well as cognitive functioning.

\section{Conflict of Interests}

The authors declare that there is no conflict of interests regarding the publication of this paper.

\section{Acknowledgments}

This work was supported by the National Natural Science Foundation of China (11232005) and the Ministry of Education Doctoral Foundation (20120074110020).

\section{References}

[1] A. A. Faisal, L. P. J. Selen, and D. M. Wolpert, "Noise in the nervous system," Nature Reviews Neuroscience, vol. 9, no. 4, pp. 292-303, 2008.

[2] E. T. Rolls and G. Deco, The Noisy Brain: Stochastic Dynamics as a Principle of Brain Function, Oxford University Press, 2010.

[3] F. Bernasconi, M. de Lucia, A. Tzovara, A. L. Manuel, M. M. Murray, and L. Spierer, "Noise in brain activity engenders perception and influences discrimination sensitivity," The Journal of Neuroscience, vol. 31, no. 49, pp. 17971-17981, 2011.

[4] T. Mori and S. Kai, "Noise-induced entrainment and stochastic resonance in human brain waves," Physical Review Letters, vol. 88, no. 21, Article ID 218101, 4 pages, 2002.

[5] T. Wennekers and G. Palm, "Syntactic sequencing in Hebbian cell assemblies," Cognitive Neurodynamics, vol. 3, no. 4, pp. 429$441,2009$.

[6] G. Werner, "From brain states to mental phenomena via phase space transitions and renormalization group transformation: proposal of a theory," Cognitive Neurodynamics, vol. 6, no. 2, pp. 203-209, 2012.

[7] G. K. Rohde, A. S. Barnett, P. J. Basser, and C. Pierpaoli, "Estimating intensity variance due to noise in registered images: applications to diffusion tensor MRI," NeuroImage, vol. 26, no. 3, pp. 673-684, 2005.

[8] D. Hunt, G. Korniss, and B. K. Szymanski, "Network synchronization in a noisy environment with time delays: fundamental limits and trade-offs," Physical Review Letters, vol. 105, no. 6, Article ID 068701, 2010.

[9] G. Deco, V. Jirs, A. R. McIntosh, O. Sporns, and R. Kötter, "Key role of coupling, delay, and noise in resting brain fluctuations," 
Proceedings of the National Academy of Sciences of the United States of America, vol. 106, no. 25, pp. 10302-10307, 2009.

[10] R. Wang, Z. Zhang, J. Qu, and J. Cao, "Phase synchronization motion and neural coding in dynamic transmission of neural information," IEEE Transactions on Neural Networks, vol. 22, no. 7, pp. 1097-1106, 2011.

[11] X. Zhang, R. Wang, Z. Zhang, J. Qu, J. Cao, and X. Jiao, "Dynamic phase synchronization characteristics of variable high-order coupled neuronal oscillator population," Neurocomputing, vol. 73, no. 13-15, pp. 2665-2670, 2010.

[12] X. Jiao and R. Wang, "Synchronous firing patterns of neuronal population with excitatory and inhibitory connections," International Journal of Non-Linear Mechanics, vol. 45, no. 6, pp. 647-651, 2010.

[13] J. Gao, J. Hu, and W. Tung, "Complexity measures of brain wave dynamics," Cognitive Neurodynamics, vol. 5, no. 2, pp. 171-182, 2011.

[14] Y. Liu, R. Wang, Z. Zhang, and X. Jiao, "Analysis of stability of neural network with inhibitory neurons," Cognitive Neurodynamics, vol. 4, no. 1, pp. 61-68, 2010.

[15] M. Hu and H. Liang, "Noise-assisted instantaneous coherence analysis of brain connectivity," Computational Intelligence and Neuroscience, vol. 2012, Article ID 275073, 12 pages, 2012.

[16] P. A. Tass, Phase Resetting in Medicine and Biology, Springer, 1999.

[17] A. Ghosh, Y. Rho, A. R. McIntosh, R. Kötter, and V. K. Jirsa, "Noise during rest enables the exploration of the brain's dynamic repertoire," PLoS Computational Biology, vol. 4, no. 10, Article ID e1000196, 2008.

[18] A. Maye, C. Hsieh, G. Sugihara, and B. Brembs, "Order in spontaneous behavior," PLoS ONE, vol. 2, no. 5, article e443, 2007.

[19] R. Wang, Z. Zhang, and X. Jiao, "Mechanism on brain information processing: energy coding," Applied Physics Letters, vol. 89, no. 12, Article ID 123903, 2006.

[20] R. Wang and Z. Zhang, "Energy coding in biological neural networks," Cognitive Neurodynamics, vol. 1, no. 3, pp. 203-212, 2007.

[21] R. Wang, Z. Zhang, and G. Chen, "Energy coding and energy functions for local activities of the brain," Neurocomputing, vol. 73, no. 1-3, pp. 139-150, 2009.

[22] E. R. Marcus and A. G. Debra, "Appraising the brain's energy budget," Proceedings of the National Academy of Sciences of the United States of America, vol. 99, no. 16, pp. 10237-10239, 2002. 


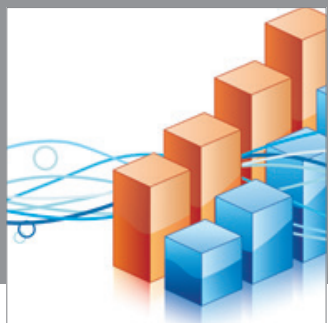

Advances in

Operations Research

mansans

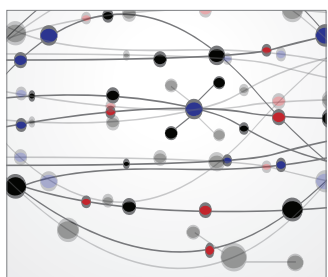

The Scientific World Journal
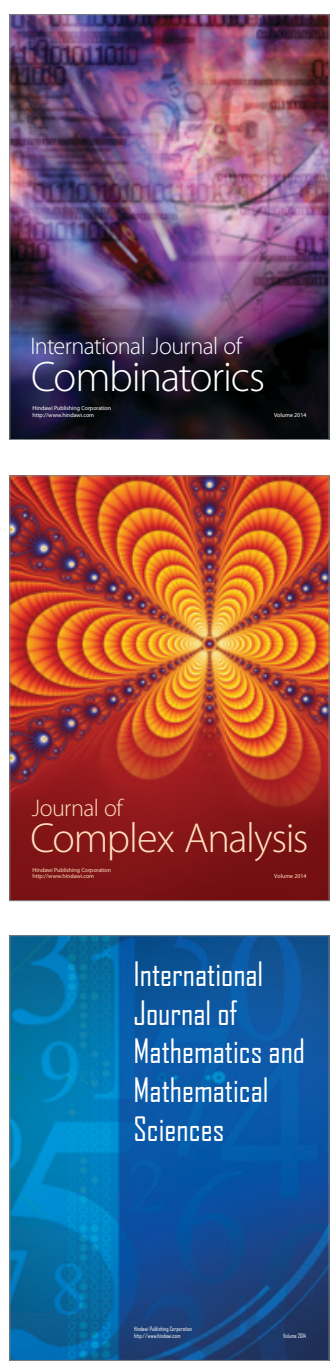
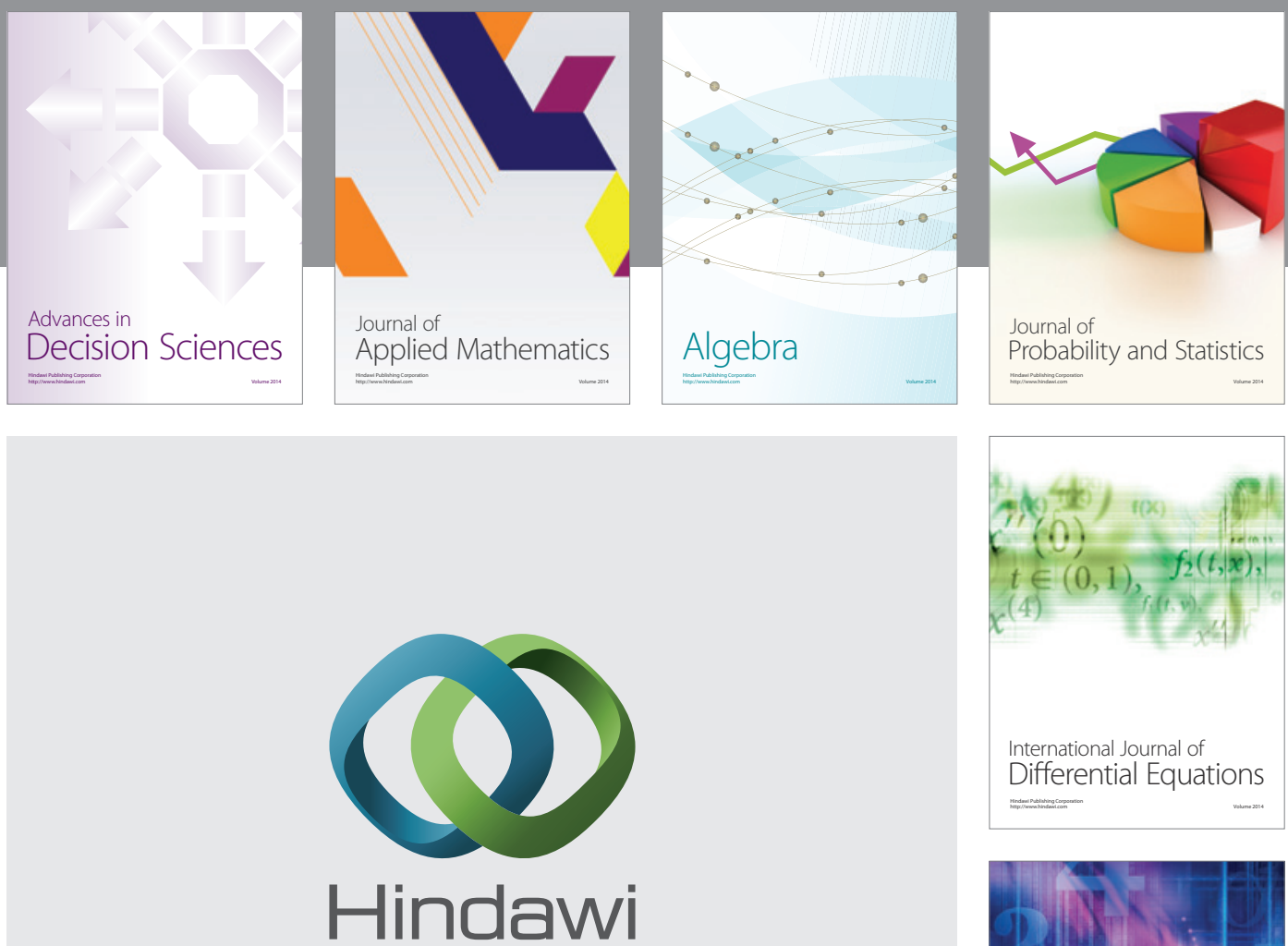

Submit your manuscripts at http://www.hindawi.com
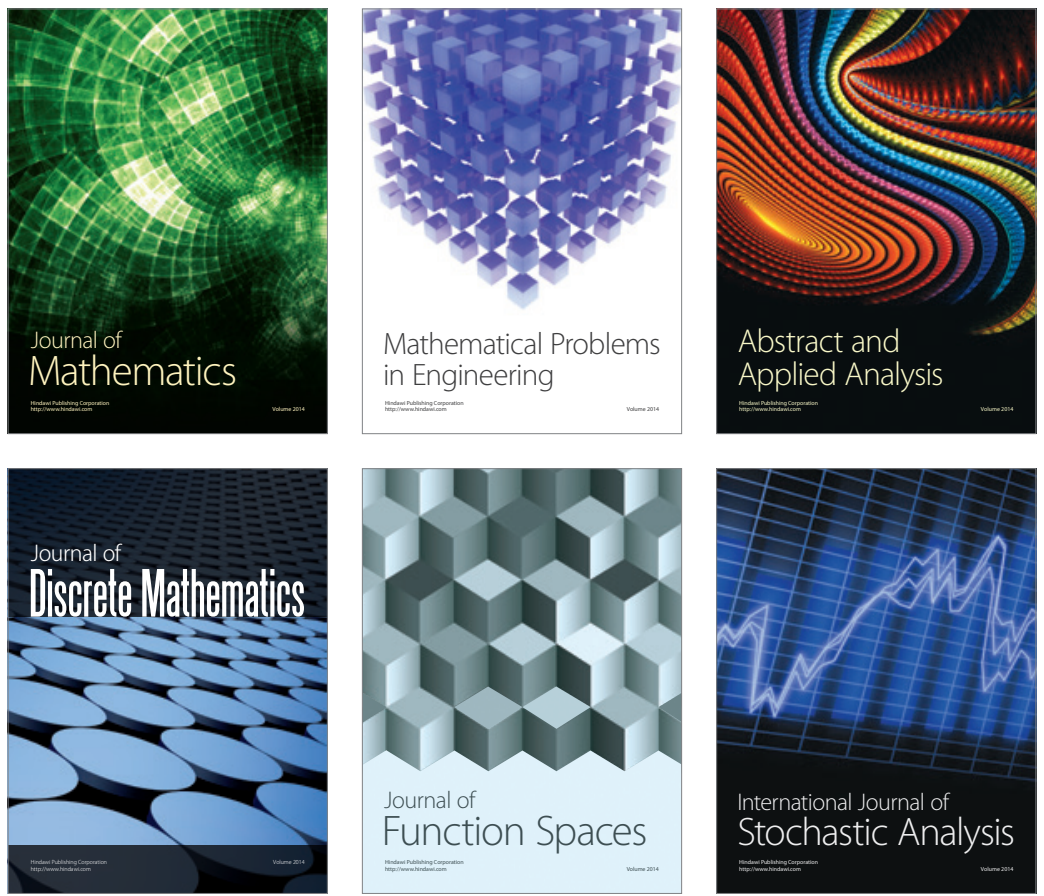

Journal of

Function Spaces

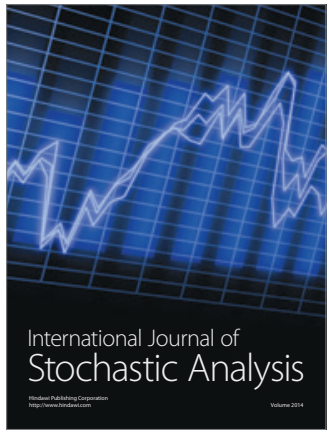

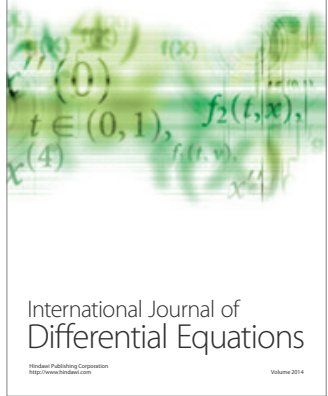
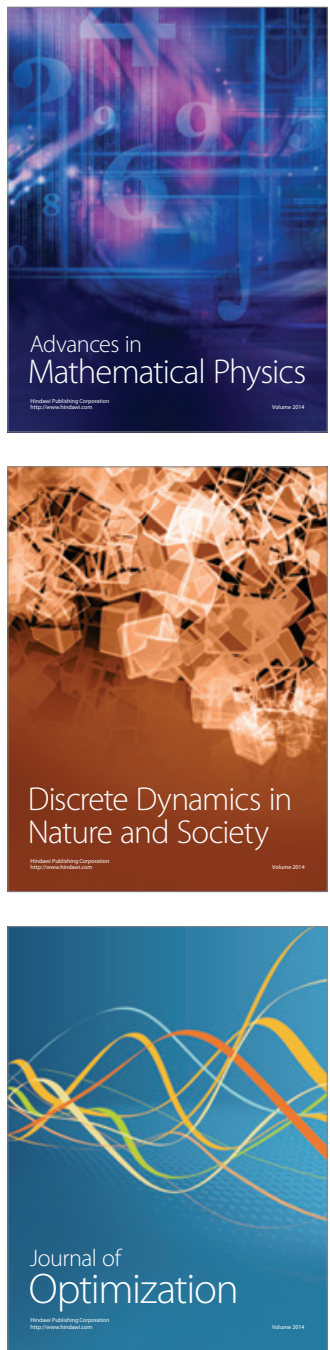